\title{
3.2 Problems of formation of the public health system in Ukraine
}

The elements of the public health system that exist in Ukraine today are inconsistent and needy optimization of existing resources and a significant increase in the efficiency of their management. Reform measures health systems have only deepened the crisis of preventive medicine. The reasons for this situation with the state of health of the population, there is a socio-economic crisis, unfavorable environmental situation, unhealthy nutrition, insufficient physical activity, high prevalence of smoking, alcohol consumption and drugs.

Public health system - a set of tools, procedures and measures implemented by governmental and non-governmental institutions to promote public health, prevent disease, increase the length of active and working age and promote a healthy lifestyle through the combined efforts of society as a whole.

Public health includes interdisciplinary approaches to epidemiology, biostatistics, and health care. Other important areas are environmental health, public health, psychohygiene, traffic safety, health economics, public policy, mental health, insurance medicine, occupational safety and health, etc.

Modern public health is the work of multidisciplinary public health teams, including physicians specializing in public health, communal medicine, infectious diseases, psychologists, epidemiologists, biostatists, medical microbiologists, and environmental health officials. public health inspectors, pharmacists, dentists, public health nurses, nutritionists and nutritionists, veterinarians, public health engineers, public health lawyers, sociologists, community development specialists, communication experts, bioethics, etc.

The concept of public health in Ukraine envisages the development of regional public health systems- creation of public health centers in each region. It is this new institution that must become regional coordinator of public health activities, to be flexible and able to fight both infectious and non-communicable diseases aimed at strengthening, protecting and preserving the health of the population, ensuring the 
definition of the social determinant of health and social justice to coordinate action call sectors of public administration.

Given the socio-political significance and economic value of individual and society -

public health, public health is considered one of the most important functions of modern state, because it is quantitative and qualitative indicators life expectancy and health of the population, a number of other indicators of social policy rich and developed countries of the world differ from the poor and underdeveloped [157]. There is so much importance to human health in the world it is important that this indicator is included in the index human development - a universal indicator level of development of society in a country.

In today's conditions, the formation and development of a public health system that would ensure the health of the population, prevent disease, increase the duration of active and working age and promote a healthy lifestyle through joint efforts is of particular importance. the whole society [161]. At the same time, the priority role should be given to the creation of an appropriate regulatory framework, adequate resource provision and modern effective management.

It is important to study the modern world experience of the functioning of public health systems, their development and improvement to justify the reform changes in Ukraine.

Population health is one of the greatest values, a necessary condition for the socio-economic development of the country. Ensuring the full life of the population, strengthening and maintaining health are among the most important goals of the world community, reflected in the principles of European policy.

The government has adopted the Concept for the Development of the Public Health System. This will help create a unified system of preventive medicine. The priority of the development of such a system is the principle that the interests of health should be taken into account in all areas of public policy. The concept defines the principles, directions, tasks, mechanisms and terms of building the public health system in order to form and implement an effective public policy to preserve and strengthen 
the health of the population. This concept defines the public health system as a set of tools, procedures and measures implemented by governmental and non-governmental institutions to strengthen the health of the population, prevent disease, increase the duration of active and working age and promote a healthy lifestyle by uniting the efforts of the whole society [161].

The public health system, as a basis for preventive medicine, should include basic measures in the field of health care and be aimed at preserving the health of the population and reducing the cost of medical care.

Ensuring the development of human resources in the field of public health requires radical changes, as there is no system of motivation, competitive selection and career growth. The current problem is the low level of wages, which makes it impossible to attract highly professional staff.

The solution of existing problems in accordance with the Concept of Public Health involves the implementation of: the creation of a multisectoral public health system with a coordinating role of the Ministry of Health; strengthening the role of the Minister of Health in the field of public health through the introduction of the principle of priority of preserving the health of the population in all areas of public authorities and defining at the legislative level the powers of the Minister to overcome threats and consequences of emergencies have a negative impact on the health of the population; ensuring the functioning. Center for Public Health of the Ministry of Health of Ukraine as a coordinator of public health programs and projects; entrusting the Center for Public Health of the Ministry of Health of Ukraine with functions to support management decisions in the field of public health, in particular to create and maintain a unified information and analytical system of medical information, methodological guidance of laboratory and analytical work of regional centers. public health.

Building a public health system in Ukraine is rapidly gaining momentum, the tasks of which are:

- cooperation with public and international organizations;

- monitoring the health of the population, assessment of social and economic factors that affect the health of the population; 
- development and implementation of a strategy for the formation of a conscious and responsible attitude of the population to their own health and personal safety;

- information support of health care institutions, education, mass media;

- creation of communication campaigns to promote the formation of a healthy lifestyle and motivate the population to maintain health;

- training of medical staff of public health institutions on public health;

- involvement of medical staff of health care institutions and public organizations in advocacy work on healthy lifestyles;

- introduction of rehabilitation systems based on technologies that provide, first of all, preservation and strengthening of health, disease prevention;

- organization of measures for the prevention of socially dangerous diseases (HIV / AIDS, tuberculosis);

- organization of immunization and surveillance programs for diseases that can be prevented by vaccination;

- prevention of infectious diseases by vaccination;

- comprehensive measures of epidemiological surveillance of infectious and non-infectious diseases, identification of risks to public health;

- coordination of activities and interaction of health care institutions regarding the collection, processing and analysis of information on the state of health of the population and resources of the health care system;

- collection and analysis of information for the formation of regional policy and strategic management in the field of public health [162].

The public health system is the basis of preventive medicine, which embodies the main systemic measures in the field of health care and aims to preserve both population and individual health of the population. Because the current system of medical care in Ukraine is not provides a preventive component, there is a need to identify new approaches and develop programs to solve problems in the field of public health and ensure the functioning of the Public Health Center of the Ministry of Health of Ukraine as a coordinator of these programs and projects. The established public health centers will ensure the preservation and strengthening of public health. 
According to the WHO, the main role in the public health system belongs to health workers. At the same time, the experience of the United States we have studied shows that volunteers and other organizations that do not require a special level of education, nurses, doctors with a bachelor's, master's or doctoral degree in public health can work in this field. $[161,163,165]$.

That is why the WHO recommends that higher education institutions organize master's, postgraduate and specialization with an emphasis on the following disciplines: global health, public health, environmental hygiene, occupational safety and health, toxicology, epidemiology, infectious diseases, health management , mental health, biostatistics, maternal and child health, nutrition, laboratory practice $[161,163$, $165]$.

Health components:

Physical health - the functioning of the human body and the state of health of each of its systems. It is important for us to know how our body should function, how to determine if we are in good health.

Mental health is our ability to receive information, our awareness of where to get the information we need, how to use it. We must be able to gather the necessary knowledge from a variety of sources and be able to use this information to make decisions about our body.

Emotional health is the ability to understand and express our feelings. Knowledge of how we feel and how others feel. Helps us express our feelings in a way that others can understand. It is important to feel comfortable, taking into account the range of emotions, and to know when people are good and when they are not.

Social health is how we feel as male or female, how we interact with other people. We need to understand what is really important to us in our relationships with friends, family, loved ones, and everyone we interact with. We need to develop skills that will help us in this relationship.

Personal health is the feeling of being a person. The feeling of developing one's "ego" ("ego" is a part of our essence that requires us to develop a sense of self and selfimprovement), is our hopes for achievement, our idea of success. Each of us finds 
unique ways of self-expression, depending on what we value and what we hope to achieve for ourselves and society. For example, some people find self-fulfillment in their work or career, others in family, and still others in activities for the benefit of others. We can determine for ourselves what brings pleasure to our own "ego".

Spiritual health is at the heart of health. Spiritual health is the core of our existence. This is the essence of our being (what we value and what is most important to us). Spirituality makes it possible to understand the relationship between the aspect of spiritual health and the other five.

Ukrainians must be provided with equal and timely access of citizens to highquality and safe blood components and preparations. The Blood Service will provide services for the procurement, processing, testing, storage and distribution of donor blood and blood components, as well as their proper clinical use. It will consist of the National Transfusion Center of the Ministry of Health, regional transfusion centers, their branches and hospital blood banks. The national register of donors, the establishment and equipping of interregional laboratory centers and blood centers with modern equipment, as well as the training of specialists, will be the next steps for the effective operation of the system.

In education, the development of public health and its further improvement will be ensured through the training and education of qualified personnel. National experts will be involved in the development and implementation of the public and postgraduate education program in the field of public health; advocacy, inclusion of the specialty "public health" in the list of fields of knowledge and specialties; and the development of a National Plan for the Development of Human Resources in the Public Health System.

And perhaps the most important area without which it is simply impossible to build an effective public health system is communication. Achieving results in the field of public health directly depends on the awareness and awareness of the population. That is why the Ministry of Health plans to develop programs of comprehensive prevention measures; to form a database on the state of health of the population and 
the environment of human life; and to conduct informational and educational campaigns to prevent disease and promote the benefits of a healthy lifestyle.

Therefore, a previous model was developed using the OneHealth Tool mental health care in Ukraine for the period from 2017 to 2030. It was calculated the cost of treatment and prevention measures, identified priority mental illnesses (depression, anxiety disorder, alcoholism, bipolar and psychotic disorders), including the cost of outpatient and primary care and resources for prevention programs (trainings), health and recreational activities.

The developed model shows that the expansion of the scale prevention and treatment of selected mental disorders provides until 2030 in total to return over UAH 4.7 million to the population of Ukraine. and prolong the duration of healthy life for 35 years. The total cost per capita will increase from $\$ 2.80$. (close 79 hryvnias) to 10.90 dollars. (about 305 hryvnias). That means for every dollar invested in prevention and improving the treatment of common mental disorders in Ukraine, we will get 2 dollars in restored productivity and added economic value.

The analysis of the data shows that the organization of public health services and their human resources differ significantly within the European region. Therefore, we should not focus on any one model of training - experts advise to work with international organizations that work closely with WHO and develop a policy of guidelines in training.

Such organizations include the Association of European Schools of Public Health (ASPHER) and the Public Health Accreditation Agency (APHEA). In particular, ASPHER has developed a list of competencies that master's graduates must achieve. To understand the needs for such staff, it is worth paying attention to the Policy Report prepared in cooperation with ASPHER, WHO and the European Observatory on Health Systems and Policies.

In connection with the above, we can conclude that in the formation of curricula should provide an increase in hours in these areas in graduation courses, as well as the inclusion in the program of sections on the study of environmental diseases, toxicology of the most commonly used and toxic chemicals. environments [162, 164, 165]. 
Achieving results in the field of public health directly depends on the awareness and awareness of the population. The main areas of further research are the development, together with the Ministry of Health of Ukraine, programs of comprehensive measures to prevent disease and promote the benefits of a healthy lifestyle, the formation of databases on public health and human environment. 